\title{
August 2018 Imaging Case of the Month: Dyspnea in a 55-Year-Old Smoker
}

\author{
Michael B. Gotway, MD \\ Department of Radiology \\ Mayo Clinic Arizona \\ Scottsdale, AZ USA
}

Clinical History: A 55-year old woman presented to the emergency room with complaints of shortness of breath and lower extremity swelling. The patient noted her shortness of breath had been worsening over the previous 6 months, especially in the last 3 months. The patient denies cough, fever, chills, and night sweats. The patient admits to some fatigue but has not lost weight recently.

The patient is a current smoker, averaging about 1 pack-per-day for the previous 40 years. Her allergies include penicillin and sulfa drugs, and her prior medical history was remarkable only for hypothyroidism and gastroesophageal reflux. Her only previous surgery was for an ectopic pregnancy. The patient's mediation list included use of opiates for pain related to a herniated disc in the lower cervical spine following an automobile accident two years earlier.

Her physical examination was unremarkable aside from obesity and mild symmetric lower extremity edema. Her vital signs were within normal limits.

Frontal and lateral chest radiography (Figure 1) was performed.

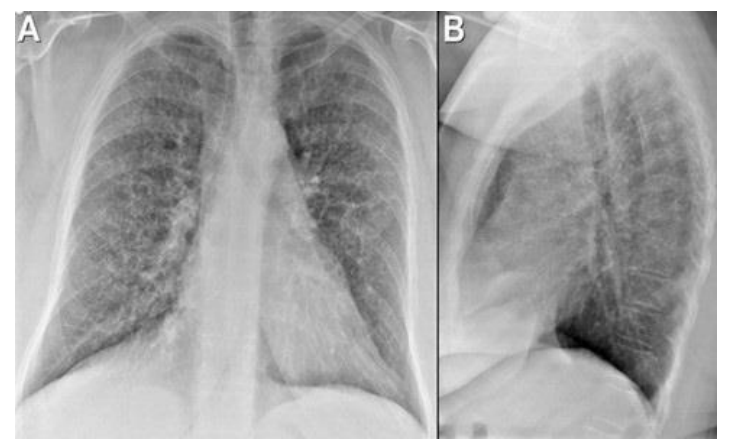

Figure 1. Frontal $(A)$ and lateral $(B)$ chest radiography.

Which of the following represents the most accurate assessment of the chest radiographic findings?

1. Chest radiography shows a mediastinal mass

2. Chest radiography shows cavitary pulmonary lesions

3. Chest radiography shows miliary nodules

4. Chest radiography shows an interstitial abnormality consisting of reticular and nodular opacities

5. Chest radiography shows basilar fibrotic opacities 


\section{Correct! \\ 4. Chest radiography shows an interstitial abnormality consisting of reticular and nodular opacities}

The frontal and lateral chest radiograph show fairly symmetric, bilateral linear, reticular, and faintly nodular opacities without a clear zonal distribution. While some nodular elements are evident, the pattern is not that of miliary disease. Some linear opacities with some faint architectural distortion may be evident, the appearance is not the basilar, peripheral or frankly subpleural reticulation associated with low lung volumes that directly suggests basal fibrotic lung disease; note that the lung volumes in Figure 1 appear relatively normal. No mediastinal mass is evident. The heart size is upper normal, but the cardiomediastinal contour is otherwise unremarkable. No evidence of cavitary lung disease is present.

At this point, which of the following represents the most appropriate step in this patient's management?

1. ${ }^{18}$ FDG-PET scan

2. Bronchoscopy with transbronchial biopsy

3. High-resolution chest CT (HRCT)

4. Comparison to prior studies

5. More than one of the above 


\section{Correct! \\ 5. More than one of the above}

The "best" answer may be comparison to prior studies, which is always the first step when evaluating an imaging abnormality. Nevertheless, high-resolution chest CT is also a reasonable choice, because the imaging findings is compelling and probably merit investigation in this symptomatic patient regardless of the chronicity as documented by comparison with prior chest radiographs. Even bronchoscopy with transbronchial biopsy is not wholly unreasonable as an interstitial abnormality associated with respiratory symptoms is present, but a tissue diagnosis is probably still premature at this point. ${ }^{18}$ FDG-PET scanning in not appropriate, however. Typically, ${ }^{18}$ FDG-PET scanning is used to investigate solitary pulmonary nodules or to stage patients with malignancies, and is not typically employed for the evaluation of interstitial lung abnormalities.

Prior chest radiography from 5 years earlier (Figure 2) was located.

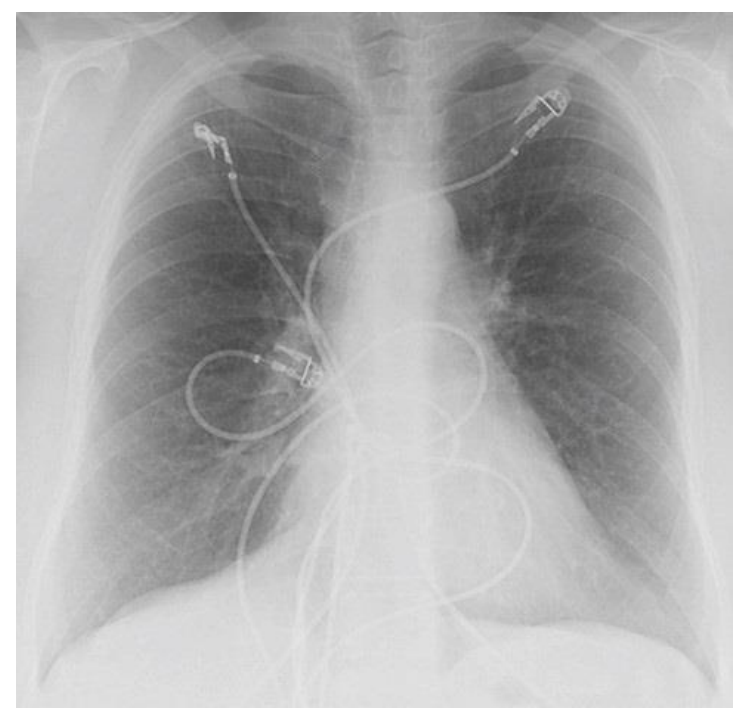

Figure 2. A comparison frontal chest radiograph performed 5 years earlier.

Which of the following represents the most accurate assessment of the current and prior chest radiographic findings?

1. Chest radiography shows that the findings seen at the presentation chest radiograph (Figure 1) are chronic and unchanged

2. Chest radiography shows that the findings seen at the presentation chest radiograph (Figure 1) are new

3. Chest radiography shows that the findings seen at the presentation chest radiograph (Figure 1) are chronic but have improved

4. Chest radiography shows that the findings seen at the presentation chest radiograph (Figure 1) have evolved from areas of consolidation previously

5. Chest radiography shows that the findings seen at the presentation chest radiograph (Figure 1) have progressed significantly 


\section{Correct! \\ 2. Chest radiography shows that the findings seen at the presentation chest radiograph (Figure 1) are new}

The chest radiograph performed 5 years earlier (Figure 2 ) is essentially normal; therefore, the abnormalities noted at the presentation chest radiograph (Figure 1) are entirely new.

The patient underwent pulmonary function testing, which showed slight reductions in the forced vital capacity and forced expiratory volume in one second, and she showed exercised-induced desaturation to $88 \%$ on room air with exercise.

At this point, which of the following represents the most appropriate step in this patient's management?

1. ${ }^{18}$ FDG-PET scan

2. Bronchoscopy with transbronchial biopsy

3. High-resolution chest CT (HRCT)

4. Thoracic MRI

5. ${ }^{68} \mathrm{Ga}$-Dotatate scan 


\section{Correct! \\ 3. High-resolution chest CT (HRCT)}

Thoracic MRI and ${ }^{18} \mathrm{FDG}-\mathrm{PET}$ scanning typically do not play a role in the evaluation of interstitial lung abnormalities. As noted above, bronchoscopy with transbronchial biopsy is not unreasonable, but is probably premature when non-invasive imaging characterization of the pulmonary process with high-resolution chest CT (HRCT) has not yet been performed. ${ }^{68} \mathrm{Ga}$-Dotatate scans are typically employed for the evaluation and staging of neuroendocrine malignancies. HRCT may provide information that allows a definitive diagnosis for the findings noted at chest radiography, or at least narrows the differential diagnostic considerations somewhat, and may be able to offer information regarding the best route for obtaining a tissue diagnosis.

The patient underwent high-resolution chest CT (Figure 3).

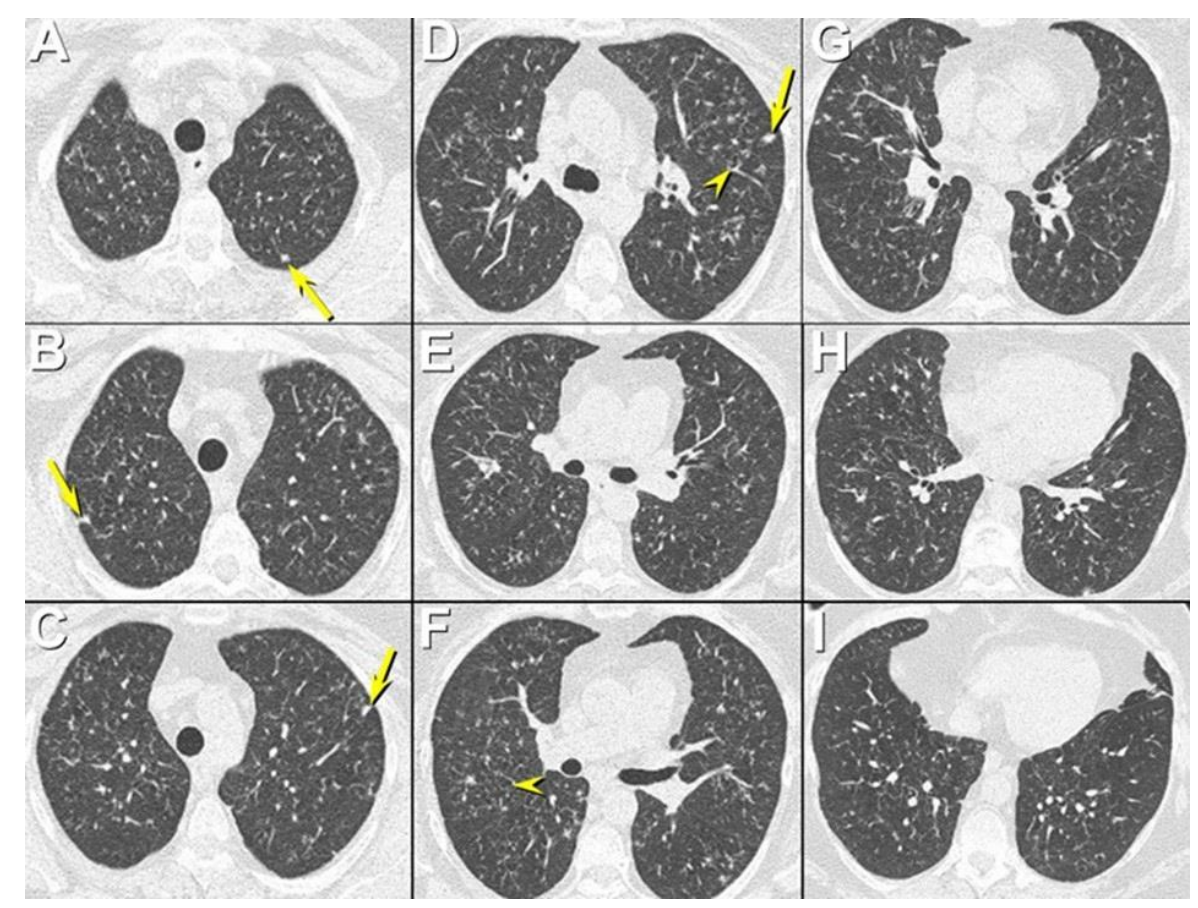

Figure 3. Representative views of axial enhanced thoracic CT displayed in lung windows.

Which of the following represents the most accurate assessment of the highresolution chest CT findings?

1. HRCT shows numerous small, somewhat randomly scattered nodules

2. HRCT shows basilar reticular abnormalities with architectural distortion, suggesting fibrotic lung disease

3. HRCT shows multifocal bronchiectasis

4. HRCT shows multifocal cavitary consolidation

5. HRCT shows multifocal ground-glass opacity 


\section{Correct!}

1. HRCT shows numerous small, somewhat randomly scattered nodules

The HRCT study shows multiple small, somewhat randomly distributed nodules, all less than $1 \mathrm{~cm}$ in size. Areas if irregular, non-septal linear and reticular opacities are also present. No clear bronchiectasis or areas of ground-glass opacity are seen. There is no evidence of consolidation, although several faintly seen foci that appear somewhat cystic are evident, but these areas are unassociated with consolidation.

Which of the following is the least appropriate consideration for the findings on this thoracic CT scan?

1. Hematogenous ("miliary") infection

2. Viral bronchopneumonia

3. Sarcoidosis

4. Langerhans cell histiocytosis

5. Lymphocytic interstitial pneumonia 


\section{Correct! \\ 1. Hematogenous ("miliary") infection}

The small nodules present are very non-specific, and, while their distribution is somewhat random, the nodules are not the typical size associated with hematogenous dissemination of infection, also often referred to as "miliary" disease. "Miliary" nodules are typically about $2 \mathrm{~mm}$ in size, far more profuse in number, and more widely distributed throughout the lungs (Figure 4), whereas the nodules in Figure 3 are far fewer in number and somewhat spare the bases.

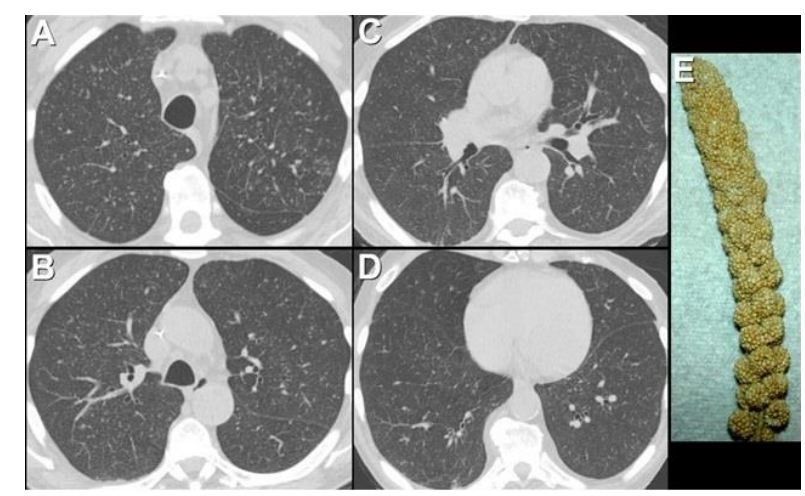

Figure 4. High-resolution chest CT (A-D) of hematogenously disseminated nodules, or "miliary" disease- numerous small nodules ranging in size from 1-3 mm equally distributed throughout the lungs (upper, mid, and lower lobes, centrally and peripherally) bilaterally. E, photograph of millet seeds.

Some of the nodules are subpleural and the nodules are upper lobe predominant, and therefore sarcoidosis does merit some consideration. Viral bronchopneumonia may present in a wide variety of ways, often as a more clear-cut bronchopneumonia pattern, but occasionally as non-specific smaller nodules. Similarly, lymphocytic interstitial pneumonia (LIP) may present in a very non-specific fashion. Typically, the diagnosis of LIP is often associated with multiple, bilateral, thin-walled cysts, but LIP may present in numerous ways, including areas of ground-glass opacity, which may be centrilobular, as mild septal thickening, or small nodules that may be perifissural or peribronchovascular in distribution; these presentations are very non-specific. Langerhans cell histiocytosis often presents as upper lobe predominant "bizarre-shaped" cysts that evolve from nodules that cavitated, so, while non-specific, the nodules on the thoracic CT in Figure 3 could be consistent with that diagnosis.

At this point, which of the following represents the most appropriate step in this patient's management?

1. Bronchoscopy with transbronchial biopsy

2. Follow-up thoracic CT

3. Upper endoscopy

4. Surgical lung biopsy

5. Pleuroscopy 


\section{Correct! \\ 1. Bronchoscopy with transbronchial biopsy}

The HRCT findings are non-specific and suggest a small nodular and interstitial abnormality that may be diagnosable with bronchoscopy with transbronchial biopsy. Surgical lung biopsy could also provide a diagnosis, but is needlessly invasive when transbronchial biopsy has not yet been performed. Follow up thoracic is unlikely to yield useful information. If the abnormalities noted at the presentation thoracic CT progress, then the need for a tissue diagnosis is reaffirmed. In contrast, a conservative approach could be pursued if the abnormalities regress, but this possibility is unlikely. Upper endoscopy could be useful for sampling mediastinal abnormalities in a periesophageal location, but no such tissue intervention targets are present. Finally, as no pleural space abnormalities are evident, pleuroscopy is not indicated.

The patient underwent bronchoscopy with transbronchial biopsy. The airways appeared normal, and no evidence of malignancy was noted on cytology review. No active inflammation was noted and no granulomas were seen. Cultures were negative, although 1+ Aspergillus species was noted, but thought to be a contaminant. The patient reported that she felt somewhat better, although her shortness of breath persisted.

At this point, which of the following represents the most appropriate step in this patient's management?

1. ${ }^{18} \mathrm{FDG}-\mathrm{PET}$

2. Repeat bronchoscopy with transbronchial biopsy and possibly lymph node biopsy with endobronchial ultrasound

3. Surgical lung biopsy

4. Serial follow up HRCT

5. CT pulmonary angiography 


\section{Correct! \\ 3. Surgical lung biopsy}

The patient underwent bronchoscopy with transbronchial biopsy and that procedure was uneventful and without complication; repeating the exam is unlikely to provide new actionable information, particularly as no clear lymphadenopathy is present. As noted previously, ${ }^{18} \mathrm{FDG}$-PET scanning is typically employed for staging malignancies and evaluating solitary pulmonary nodules, and not for the investigation of interstitial abnormalities. The absence of tracer utilization within the lung parenchymal abnormalities at ${ }^{18}$ FDG-PET scanning in this patient would not obviate the need for a tissue diagnosis, whereas the presence of tracer uptake within the pulmonary abnormalities would merely reaffirm the need for such; therefore, ${ }^{18} \mathrm{FDG}$-PET scanning is unlikely to provide management-altering information for this patient. Repeating the $\mathrm{HRCT}$, or performing a repeat thoracic CT using pulmonary angiographic technique, would also probably not provide useful information in a patient with multifocal interstitial abnormalities and significant respiratory symptoms; indeed, the lung parenchymal findings are unlikely to be the result of thromboembolic disease. At this point, surgical lung biopsy is an indicated procedure.

The patient underwent repeat chest CT (Figure 5).

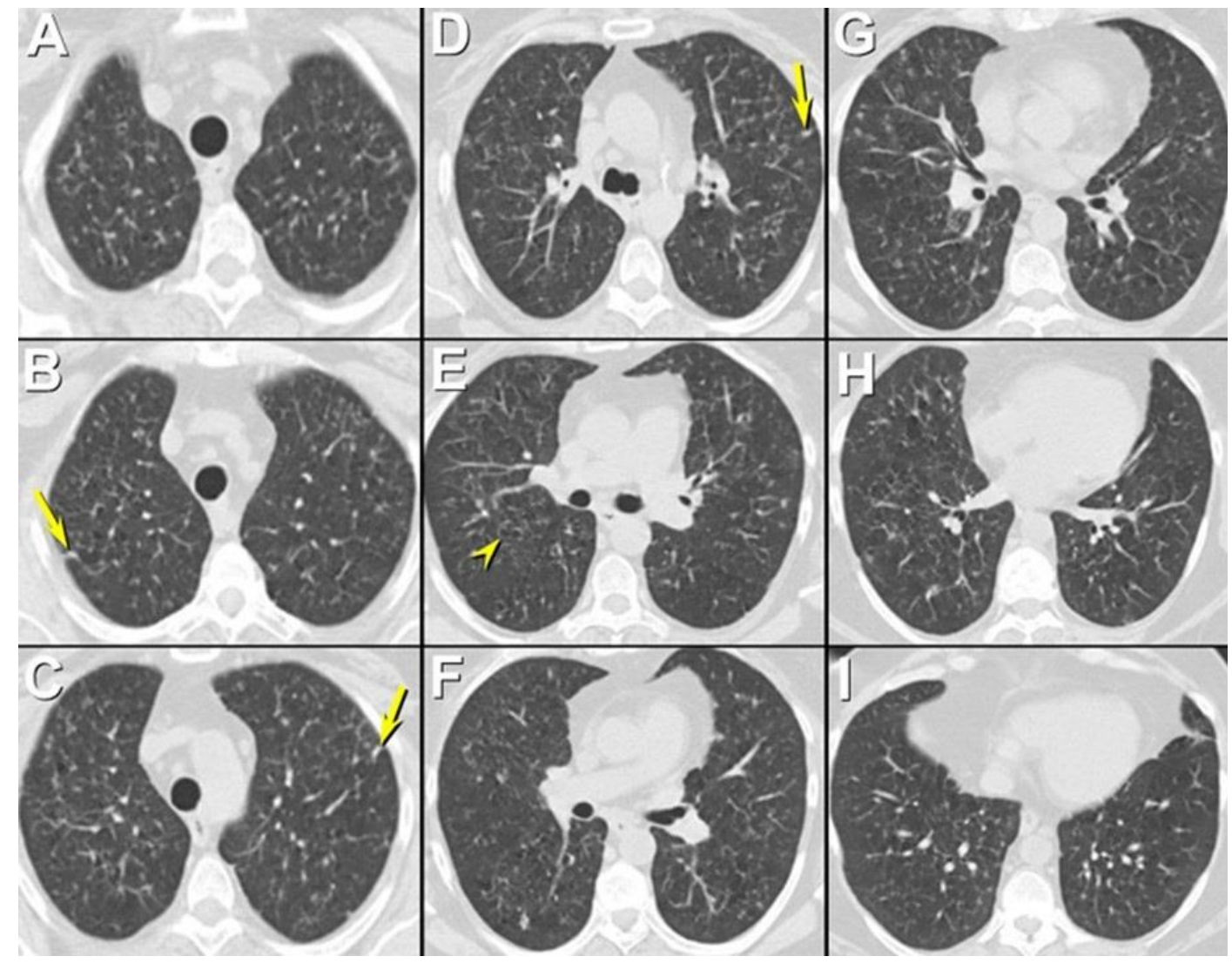

Figure 5. Axial enhanced thoracic CT displayed in lung windows performed 1 month after the presentation thoracic CT (Figure 3). 
Which of the following represents the most accurate assessment of the repeat highresolution chest CT findings?

1. The repeat HRCT shows numerous small, somewhat randomly scattered nodules unchanged from the previous thoracic CT (Figure 3)

2. The repeat HRCT shows worsening of the nodular and cystic abnormalities seen at the presentation thoracic CT (Figure 3)

3. The repeat HRCT shows improvement in the nodular and cystic abnormalities seen at the presentation thoracic CT (Figure 3)

4. The repeat HRCT shows new mediastinal and peribronchial lymph node enlargement

5. The repeat HRCT shows development of new multifocal ground-glass opacity and consolidation 


\section{Correct!}

\section{The repeat HRCT shows numerous small, somewhat randomly scattered nodules unchanged from the previous thoracic CT (Figure 3)}

The patient was referred to cardiothoracic surgery for surgical lung biopsy. Videoassisted thoracoscopic lung biopsy was attempted, but, owing to technical difficulties and inability to obtain adequate one-lung ventilation, was converted to an open thoracotomy for lung biopsy. Histopathological material obtained from lingular biopsy showed active smoking-related changes with cysts and stellate nodules consistent with both active and inactive Langerhans cell histiocytosis.

Diagnosis: Pulmonary Langerhans Cell Histiocytosis

\section{References}

1. Seaman DM, Meyer CA, Gilman MD, McCormack FX. Diffuse cystic lung disease at high-resolution CT. AJR Am J Roentgenol. 2011; 196(6):1305-11. [CrossRef] [PubMed]

2. Seely JM, Salahudeen S Sr, Cadaval-Goncalves AT, Jamieson DH, Dennie CJ, Matzinger FR, Souza CA. Pulmonary Langerhans cell histiocytosis: a comparative study of computed tomography in children and adults. J Thorac Imaging. 2012;27(1):65-70. [CrossRef] [PubMed]

3. Sundar KM, Gosselin MV, Chung HL, Cahill BC. Pulmonary Langerhans cell histiocytosis: emerging concepts in pathobiology, radiology, and clinical evolution of disease. Chest. 2003;123(5):1673-83. [CrossRef] [PubMed]

4. Vassallo R, Harari S, Tazi A. Current understanding and management of pulmonary Langerhans cell histiocytosis. Thorax. 2017;72(10):937-45. [CrossRef] [PubMed]

5. Gupta N, Vassallo R, Wikenheiser-Brokamp KA, McCormack FX. Diffuse cystic lung disease. part I. Am J Respir Crit Care Med. 2015;191(12):1354-66. [CrossRef] [PubMed]

6. Gupta N, Vassallo R, Wikenheiser-Brokamp KA, McCormack FX. Diffuse cystic lung disease. part II. Am J Respir Crit Care Med. 2015;192(1):17-29. [CrossRef] [PubMed]

7. Vassallo R, Ryu JH. Pulmonary Langerhans' cell histiocytosis. Clin Chest Med. 2004; 25(3):561-71. [CrossRef] [PubMed] 